\title{
Identification of active release planes using ground-based differential InSAR at the Randa rock slope instability, Switzerland
}

\author{
V. Gischig ${ }^{1}$, S. Loew ${ }^{1}$, A. Kos ${ }^{1}$, J. R. Moore ${ }^{1}$, H. Raetzo ${ }^{2}$, and F. Lemy ${ }^{1, *}$ \\ ${ }^{1}$ Geological Institute, Swiss Federal Institute of Technology (ETH), Zürich, Switzerland \\ ${ }^{2}$ Federal Office for the Environment (FEON), Bern, Switzerland \\ *now at: Federal Agency for Nuclear Control (FANC), Brussels, Belgium
}

Received: 10 September 2009 - Accepted: 10 November 2009 - Published: 4 December 2009

\begin{abstract}
Five ground-based differential interferometric synthetic aperture radar (GB-DInSAR) surveys were conducted between 2005 and 2007 at the rock slope instability at Randa, Switzerland. Resultant displacement maps revealed, for the first time, the presence of an active basal rupture zone and a lateral release surface daylighting on the exposed 1991 failure scarp. Structures correlated with the boundaries of interferometric displacement domains were confirmed using a helicopter-based LiDAR DTM and oblique aerial photography. Former investigations at the site failed to conclusively detect these active release surfaces essential for kinematic and hazard analysis of the instability, although their existence had been hypothesized. The determination of the basal and lateral release planes also allowed a more accurate estimate of the currently unstable volume of $5.7 \pm 1.5$ million $\mathrm{m}^{3}$. The displacement patterns reveal that two different kinematic behaviors dominate the instability, i.e. toppling above $2200 \mathrm{~m}$ and translational failure below. In the toppling part of the instability the areas with the highest GB-DInSAR displacements correspond to areas of enhanced micro-seismic activity. The observation of only few strongly active discontinuities daylighting on the 1991 failure surface points to a rather uniform movement in the lower portion of the instability, while most of the slip occurs along the basal rupture plane. Comparison of GB-DInSAR displacements with mapped discontinuities revealed correlations between displacement patterns and active structures, although spatial offsets occur as a result of the effective resolution of GBDInSAR. Similarly, comparisons with measurements from total station surveys generally showed good agreement. Discrepancies arose in several cases due to local movement of blocks, the size of which could not be resolved using GBDInSAR.
\end{abstract}

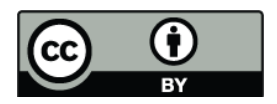

Correspondence to: V. Gischig (valentin.gischig@erdw.ethz.ch)

\section{The Randa rockslope instability}

In the spring of 1991 two large rockslides occurred above the village of Randa in the Matter Valley (southern Swiss Alps). Within a three week period, a total volume of 30 million $\mathrm{m}^{3}$ of rock was released. The resulting talus cone dammed the river and formed a lake, which flooded the nearby village (Schindler et al., 1993). The kinematics and mechanisms of the 1991 events were studied by Sartori et al. (2003) and Eberhardt et al. (2004). One legacy of these failures was the formation of an $800 \mathrm{~m}$ high scarp, behind which an estimated 3-9 million $\mathrm{m}^{3}$ (Ischi et al., 1991) of rock remains unstable. In 1995, a geodetic network, consisting of seven 3-D reflectors and eighteen 1-D reflectors located in the crown and along the edge of the scarp, was setup to monitor rock mass displacements (Jaboyedoff et al., 2004). The kinematics of the current instability was investigated in a research project begun in 2001, which included field mapping and borehole logging (Willenberg et al., 2008a), geophysical imaging of the 3-D extent of discontinuities (Heincke et al., 2005 and 2006; Spillmann et al., 2007a), monitoring of micro-seismic activity associated with rock mass deformation (Spillmann et al., 2007b), as well as surface crack and borehole monitoring (Willenberg et al., 2008b). Displacements resolved from total station measurements were used to approximate the extents of the moving mass. However, due to their sparse distribution, large uncertainties remained. The deformation pattern was interpreted as block toppling in the upper part of the instability (Loew et al., 2007). A basal rupture surface was postulated by Jaboyedoff et al. (2004) by interpreting geodetic data and later by Willenberg et al. (2008b). However, it could not be detected with geophysical imaging or within a $120 \mathrm{~m}$ deep borehole. All previous investigations were limited to the accessible area at the top of the instability, whereas large portions of the currently unstable rock mass could not be studied due to the inaccessibility of the nearly $800 \mathrm{~m}$ high cliff face. Inspection of photographs of the 1991

Published by Copernicus Publications on behalf of the European Geosciences Union. 
failure surface could not conclusively prove the existence of a basal rupture plane or other active structures that may outcrop on the scarp, since the pattern of faults and joints is too complex and no in-situ monitoring is available to confirm movements.

In this manuscript we present data from five ground-based differential InSAR (GB-DInSAR) surveys carried out between September 2005 and September 2007. We first describe relevant technical details about the technique, the surveys at Randa and the processing of the data. Displacement maps obtained from these surveys are then interpreted towards the observation of active large-scale release structures bounding the instability, which have remained undetected by previous investigations. The datasets are also compared to the structural map by Willenberg et al. (2008a) and to results from complementary geodetic surveys and microseismic monitoring. Observations are discussed in terms of their implications for kinematics of the rock slope instability, as well as with regards to the spatial and temporal resolution limitations of the technique.

\section{Ground-based differential radar interferometry}

\subsection{The GB-DInSAR technique}

Ground-based synthetic aperture radar interferometry is an established, reliable method for spatial displacement monitoring of rock slopes (e.g. Tarchi et al. 2003a, b; Lingua et al., 2008), and is especially valuable when inaccessibility prohibits the application of other traditional monitoring techniques. For ground-based systems, the synthetic aperture radar (SAR) method relies on linear translation of an antenna-transmitter pair to create a synthetic aperture (i.e. an antenna length in the range of $2.0-2.7 \mathrm{~m}$ ). A series of observations are combined in post-processing as if they had been made simultaneously using a large antenna, providing the resolution in azimuth (Tarchi et al., 2003a, b). The resulting SAR image is a map of complex numbers containing both the amplitude and the phase of the radar signals reflected by the target. The range resolution cell size of such images is inversely proportional to the frequency bandwidth of the system, whereas the azimuthal resolution is inversely proportional to the synthetic aperture length. Two subsequent SAR images can be combined to create an interferogram (i.e. a GB-DInSAR image) by extracting the phase difference $\Delta \varphi$ between the two acquisitions from the complex values of each resolution cell. The corresponding displacement $(\Delta s)$ is then obtained as:

$\Delta s=\frac{\Delta \varphi}{4 \pi} \lambda$

where $\lambda$ is the wavelength of the signals. An important property of the GB-DInSAR method is the ambiguity of the phase differences, which can only vary between $\pm \pi$. Thus, displacements smaller or larger than $\pm \lambda / 4$ result in apparent val- ues between $\pm \lambda / 4$ differing from the real value by a multiple of the full wavelength. This effect is called phase wrapping and is important for interpretation of GB-DInSAR displacement maps. The reliability of GB-DInSAR data is controlled by both the reflectivity of the ground and temporal decorrelation between acquisitions. Reflectivity of a surface determines the strength of the signal reflected back to the receiver by the target, i.e. the reflected power. It is generally low for densely vegetated areas and smooth targets not perpendicular to the line-of-sight (LOS). Decorrelation results from strong movements within a resolution cell between two acquisitions, e.g. due to unstable debris cover or differential displacements. A measure of the strength of decorrelation is the signal coherence from two subsequent acquisitions, which is defined as:

$$
\gamma=\frac{E\left[m s^{*}\right]}{\sqrt{E[m]^{2} E[s]^{2}}}
$$

where $m$ and $s$ are the complex numbers of two acquisitions for one particular resolution cell, $(*)$ denotes complex conjugate, and $E$ signifies the expectation value. The signal coherence is a number between 0 and 1 and is a measure of the similarity of the transmitted and the received signal. In an interferogram, unreliable data can be masked by setting a threshold for both reflected power and coherence. Further processing steps are commonly performed on GB-DInSAR images, e.g. multi-look filtering (Lee et al., 1994) and other filters, which often lower the theoretical spatial resolution of the system since neighboring cells are no longer independent.

\subsection{GB-DInSAR data acquisition and processing at the Randa instability}

The 1991 failure surface of the Randa rock slope instability is well suited for application of GB-DInSAR due to the absence of vegetation and the possibility to align the radar such that the LOS is sub-parallel to the rock mass displacements. Between 2005 and 2007, five GB-DInSAR measurements were conducted by Ellegi Srl (Milano, Italy) using their GB-DInSAR system called LiSA. The LiSA base station was located at $1560 \mathrm{~m}$ a.s.l. on the valley wall opposite the 1991 failure scarp. System specifications and acquisition parameters for these radar surveys are summarized in Table 1, while campaign dates and time intervals are shown in Table 2. Methods employed in processing radar images are described by Fortuny and Sieber (1994) and Tarchi et al. (2003a, b), while some relevant points concerning the Randa datasets are described here. Since Ellegi Srl also processed the Randa data using their proprietary methods, we can only give a basic description of the processing steps.

To exclude unreliable phase measurements, the thresholds for coherence and reflected power given in Table 1 were used. The radar data were gridded on a regular $2 \times 2 \mathrm{~m}$ Cartesian grid (Leva et al., 2003), which is smaller than the theoretical resolution of the system $(1.9 \times 4-6.5 \mathrm{~m}$, Table 1$)$. This 
Table 1. Acquisition parameters for the GB-DInSAR surveys.

\begin{tabular}{lr}
\hline Target distance & $1.3-2 \mathrm{~km}$ \\
Range resolution & $1.9 \mathrm{~m}$ \\
Minimal azimuthal resolution & $4 \mathrm{~m}$ \\
Maximum azimuthal resolution & $6.5 \mathrm{~m}$ \\
Synthetic aperture length & $2 \mathrm{~m}$ \\
Frequency range & $17.10-17.18 \mathrm{GHz}$ \\
Coherence and power (reflectivity) threshold & 0.65 and $-50 \mathrm{~dB}$ \\
\hline
\end{tabular}

Table 2. Dates of the GB-DInSAR surveys and time intervals to the previous survey.

\begin{tabular}{lcr}
\hline Survey & Date & $\begin{array}{r}\text { Time to previous } \\
\text { survey }\end{array}$ \\
\hline Reference & 22 September 2005 & \\
1st repeat (image B) & 20 November 2005 & 59 days \\
2nd repeat (image C) & 3 October 2006 & 316 days \\
3rd repeat (Fig. 8) & 7 June 2007 & 248 days \\
4th repeat & 26 September 2007 & 111 days \\
\hline
\end{tabular}

interpolation is used to prevent aliasing during later processing, but also means that the images cannot be interpreted on the level of single pixels. A proprietary multi-look filter was used to help reduce phase noise due to the speckle effect (Lee et al., 1994), i.e. a number of independent views of the scene were averaged to smooth the grainy character of nonaveraged views. Other proprietary post-processing steps, the details of which are not available to the authors, involve filters running over $7 \times 7$ pixels in the case of the Randa acquisitions. Thus, the resulting images have an effective resolution of $14 \times 14 \mathrm{~m}$. Values of single pixels are therefore not independent of their neighboring pixels, and thus differential displacement patterns can only be interpreted if they are consistent over a distance greater than the effective resolution. Finally, the images were overlain on a hill-shaded DTM with resolution of $0.5 \mathrm{~m}$, which was derived from helicopter-based LiDAR data (acquired by Helimap Systems SA).

\section{Results}

\subsection{Displacement maps}

GB-DInSAR image maps showing displacements between September and November 2005 (59 days), as well as between November 2005 and October 2006 (316 days) are presented in Fig. 1. The color scale shows displacements from -4.4 to $+4.4 \mathrm{~mm}$ corresponding to $\pm \lambda / 4$ of the radar. Negative values (red to yellow) show displacement along the LOS towards the observer, positive values (light blue to blue) away from the observer, and turquoise values represent zero displacement.
An overview of the GB-DInSAR scene including the base station position, the line-of-sight, as well as the locations of detail figures described later is given in Fig. 2 (displacement image between October 2006 and June 2007; 248 days). All displacement maps reveal a similar overall pattern. In the talus at the bottom of the images (region (a) in Fig. 1), measured displacements suffer from decorrelation and phase wrapping (displacements $>\lambda / 4$ ). Displacements on image B (59 days interval), however, show a consistent pattern interpreted as consolidation of the debris cone (Fig. 3). On the same image, an elongated decorrelation pattern close to the steep orthogneiss cliff on the debris cone correlates with active debris flow channels. The steep orthogneiss cliff just above the talus (region b) is stable throughout all measurements. Above this stable cliff is a sharp transition to unstable rock (region d), which is best observed on image C (316 days interval). In region (d), displacements are relatively uniform over a large area. Slightly higher displacements in this region result in phase wrapping on image $\mathrm{C}$, i.e. sudden transitions between negative (red) and positive (blue) displacements. To the south of region (d), a large portion of the 1991 scarp is covered with debris (region c). Decorrelation due to debris movement limits interpretation in this area. Region (e) delineates a portion of the scarp which is primarily stable, although some blocks moving towards the scarp edge can be seen. Above region (d) and to the north of the stable region (e), displacements increase towards the top of the scarp (region f). Here, the greatest displacements were measured. The transition from the uniformly moving region (d) to region (f) occurs at about $2200 \mathrm{~m}$. This transition is clearly visible on image $B$, whereas image $C$ suffers from phase wrapping. Above region (f), some data gaps occur in grassy areas due to decorrelation and shadowing. In the upper part of the images (region g) extended stable areas are found.

\subsection{Release planes identified on GB-DInSAR displacement maps}

The lower boundary of the unstable rock mass can be identified by a sharp transition from $0 \mathrm{~mm}$ displacement (region $\mathrm{b}$ ) to $4 \mathrm{~mm}$ displacement (region d) on image $\mathrm{C}$ in Fig. 1 (enlarged in Fig. 4). The magnitude of the displacement rate and the location of the transition are consistent across all images. The location of this transition corresponds to the trace of the boundary between the two primary lithologies on the rock slope: strongly fractured paragneiss and schist overlaying more competent orthogneiss. However, the lithological boundary is sub-parallel to foliation and dips into the slope at roughly $20^{\circ}$. Water discharge is often observed along this boundary after snow melt and heavy rainfall. This structure can also be identified on high resolution aerial photographs (Fig. 4). The transition between the stable cliff and the instability above is characterized by a sharp increase in displacement over one pixel, or by decorrelation across 2-3 pixels. Bearing in mind that the original range resolution before 


\section{Displacement along LOS}
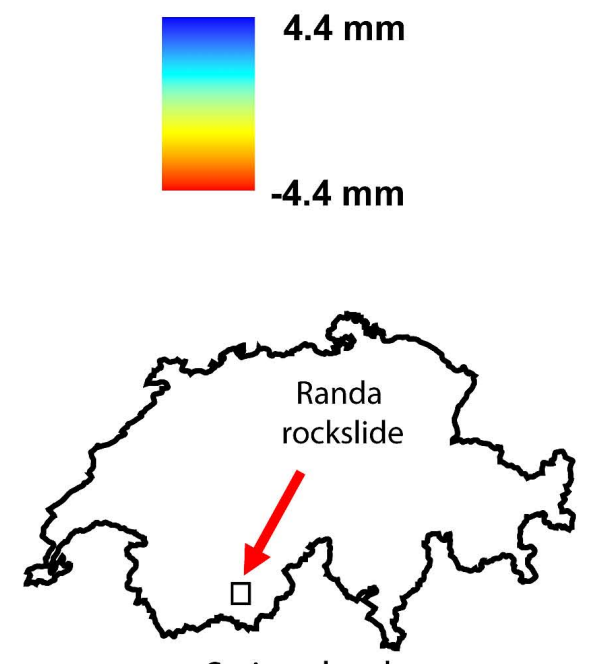

Switzerland
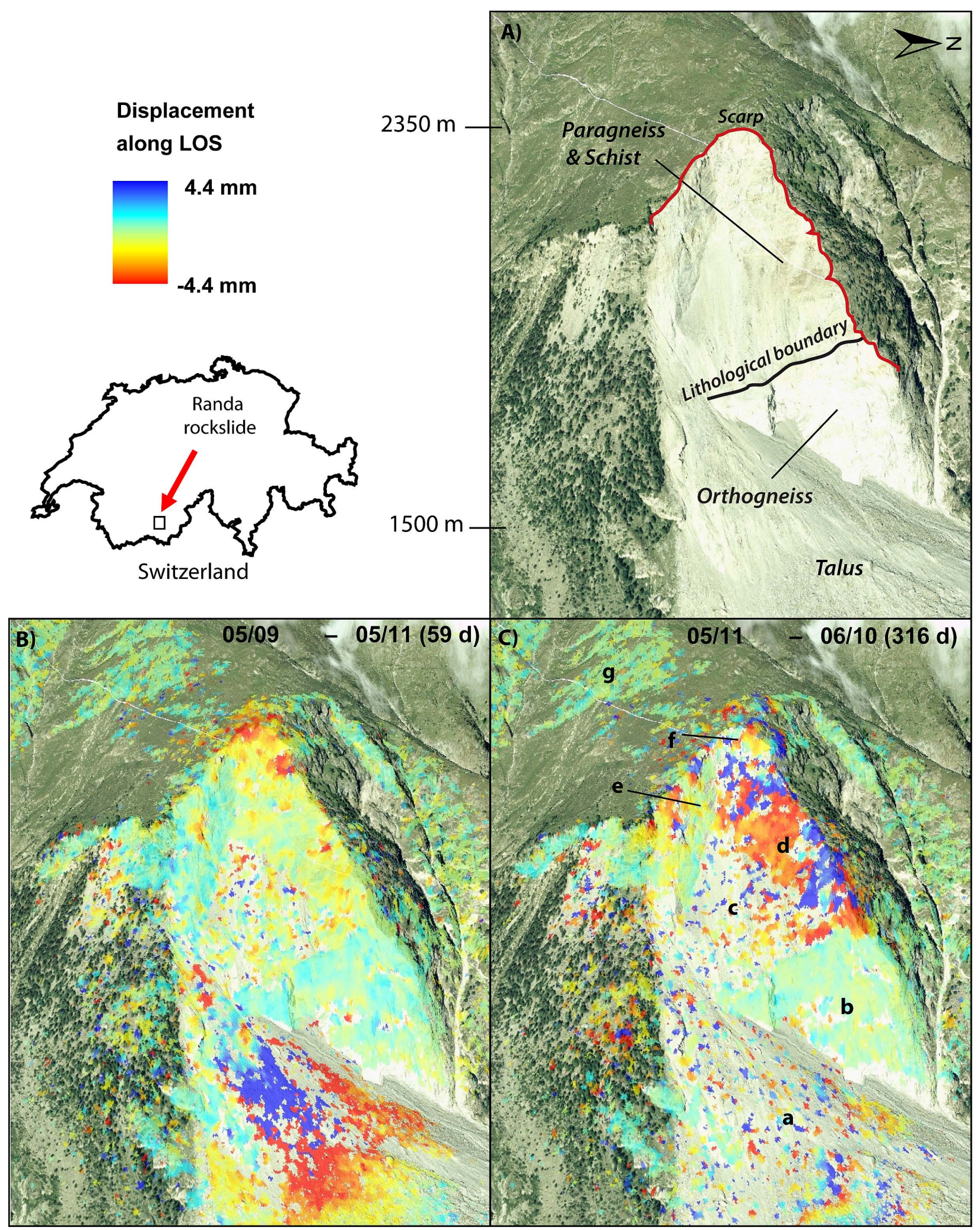

Fig. 1. (A) Orthophoto draped on DTM. The overview scene is the view of an observer at about 2000 m looking approximately towards NW. (B) GB-DInSAR displacement map derived from the 1st repeat with respect to the reference survey. (C) Displacement map from the 2 nd with respect to the 1 st repeat survey $(\mathrm{C})$. Dates $(\mathrm{yy} / \mathrm{mm})$ of the surveys, as well as the number of days between successive surveys are shown. 


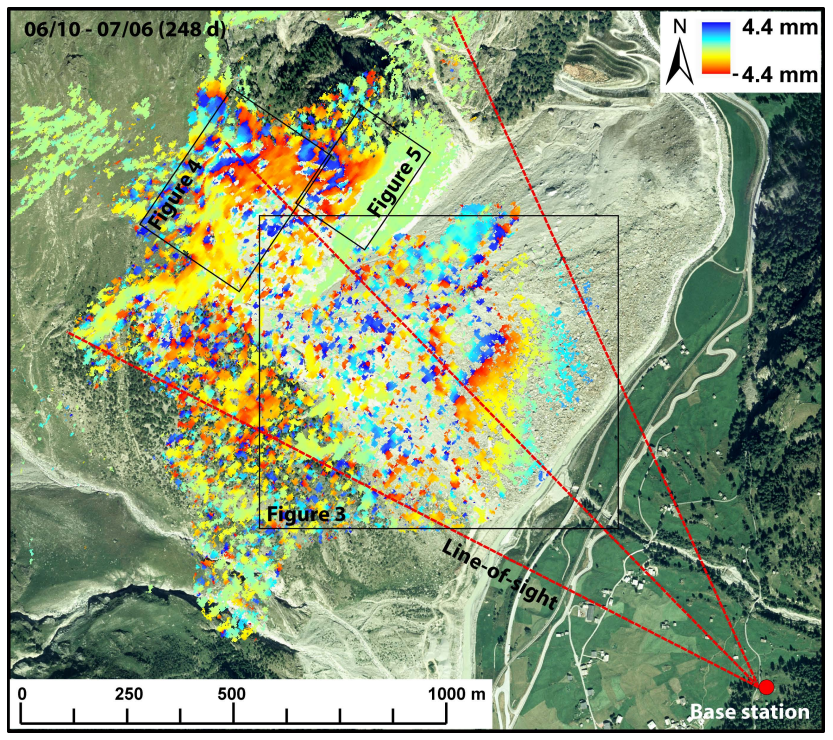

Fig. 2. Overview map showing the locations of Figs. 3, 4, and 5 overlain on an orthoimage. GB-DInSAR displacement map is derived from the 3 rd with respect to the 2 nd repeat survey ( 248 days). Also shown is the line-of-sight and location of the base-station on the opposite valley flank.

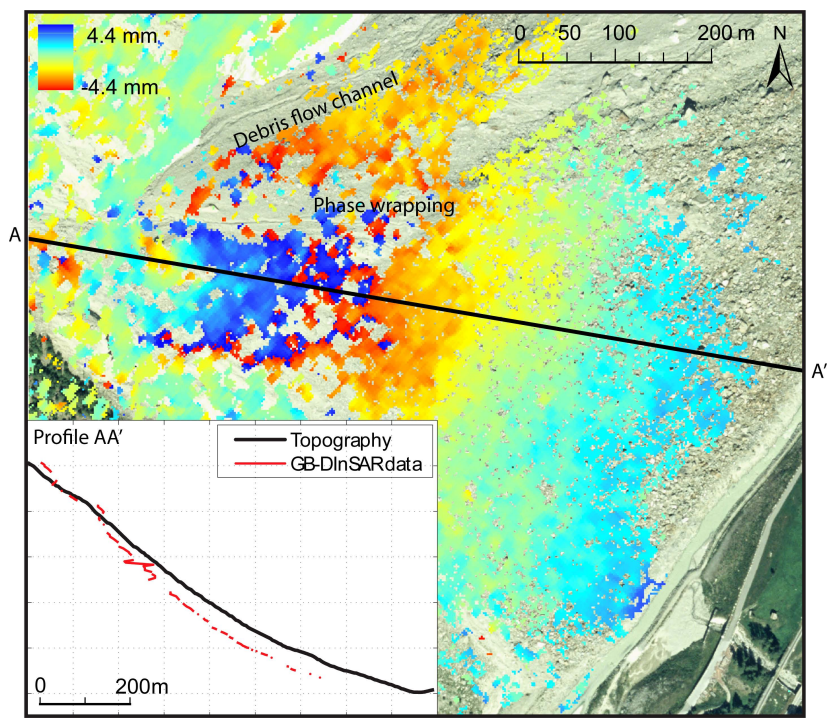

Fig. 3. 59 days interval GB-DInSAR image showing consolidation of the debris cone. The abrupt change from negative (red) to positive (blue) values is caused by phase wrapping. For profile AA' the GB-DInSAR values were phase unwrapped and presented as a 10000 times exaggerated change in topography (red line). See Fig. 2 for location of this detail figure.

interpolation is about $2 \mathrm{~m}$ wide, this decorrelation pattern can be interpreted as differential displacement causing loss of coherence. This indicates that deformation is concentrated at the base of the instability within one resolution cell, or a nar- row zone only a few meters wide. GB-DInSAR displacement maps also indicate that the transition zone has a minimum length of $150-200 \mathrm{~m}$. We interpret this feature as the basal rupture zone of the instability.

The southern boundary of the instability can also be identified on the GB-DInSAR displacement maps (Fig. 5). It is characterized by a sub-vertical, sharp transition between $0 \mathrm{~mm}$ displacement in the south (region (e) in Fig. 1) and a displacement pattern in the north that shows increasing displacements from 4 to $12 \mathrm{~mm}$ to the top of scarp (region (f) in Fig. 1). Note that the apparent positive values result from phase wrapping. High resolution photographs and the LiDAR DTM show that this lateral boundary coincides with a fault surface oriented $095 / 70$, containing striations that dip towards the valley. The surface also corresponds to a lateral release plane from the 1991 rockslides. According to the displacement maps, the stratum underlying the fault is stable although some isolated toppling blocks near the scarp can be identified. To the north, the overlying unstable stratum is composed of blocky and highly fractured rock. The intersection of this plane with the ground surface shows that it matches well with steeply dipping, NS-striking faults mapped by Willenberg et al. (2008a). We conclude that this fault corresponds to the southern release plane of the current instability.

\subsection{Active structures within the instability}

A more detailed analysis of deformation patterns within the instability was carried out in order to identify additional active structures critical for kinematic analysis, as well as to explore the resolution limits of the GB-DInSAR technique in structural analyses. The GB-DInSAR displacement maps were first overlain with a map of large-scale discontinuities (faults and fracture zones) obtained from extensive field investigations in the accessible part of the 1991 rockslide crown (Willenberg et al., 2008a) (Fig. 6). The discontinuities are drawn in black if there are corresponding displacement patterns noted on the GB-DInSAR maps (such as steps in the displacement or elongated patterns of decorrelation) and in grey otherwise. In case of correlation between GB-DInSAR displacement patterns and mapped discontinuities, the alignment is usually not perfect but often offset by a few pixels (Fig. 6). Mostly discontinuities which belong to a set striking nearly perpendicular to the LOS of the GB-DInSAR show correlation. This is expected since such discontinuities usually have the largest differential displacement component along the LOS. The opening rates of discontinuities Z1 and Z9 have been monitored with periodic hand measurements over the last 7 years, and are $3.5 \mathrm{~mm} / \mathrm{yr}$ and $2.3 \mathrm{~mm} / \mathrm{yr}$, respectively. Opening rates for these discontinuities were also estimated from the GB-DInSAR data by extracting displacements from both sides of the cracks from all time intervals. The time series' derived reveal opening rates of $2.7 \pm 0.6 \mathrm{~mm} / \mathrm{yr}$ for $\mathrm{Z} 1$ and $1.9 \pm 0.6 \mathrm{~mm} / \mathrm{yr}$ for $\mathrm{Z} 9$, 


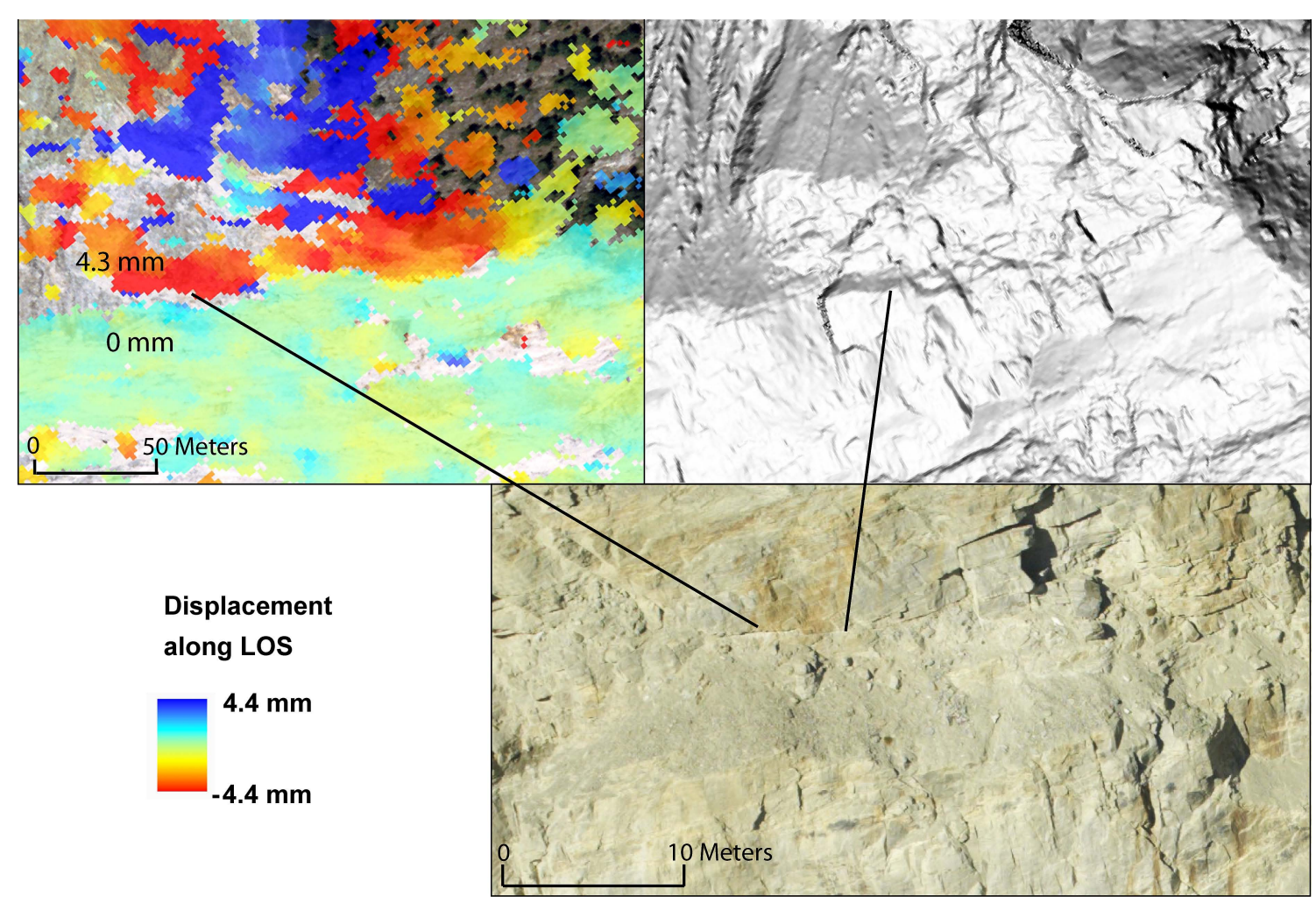

Fig. 4. Oblique aerial view of GB-DInSAR displacement map (316 days interval; top left), shaded DEM from helicopter-based LiDAR ( $50 \mathrm{~cm}$ resolution; top right), and high resolution photo (pixel size $\sim 2-3 \mathrm{~cm}$; bottom right) showing the basal rupture zone. The displacement pattern indicates an abrupt change from 0 to $-4.3 \mathrm{~mm}$ along LOS. This zone coincides with a lithological boundary on the rock face. Location is shown in Fig. 2.

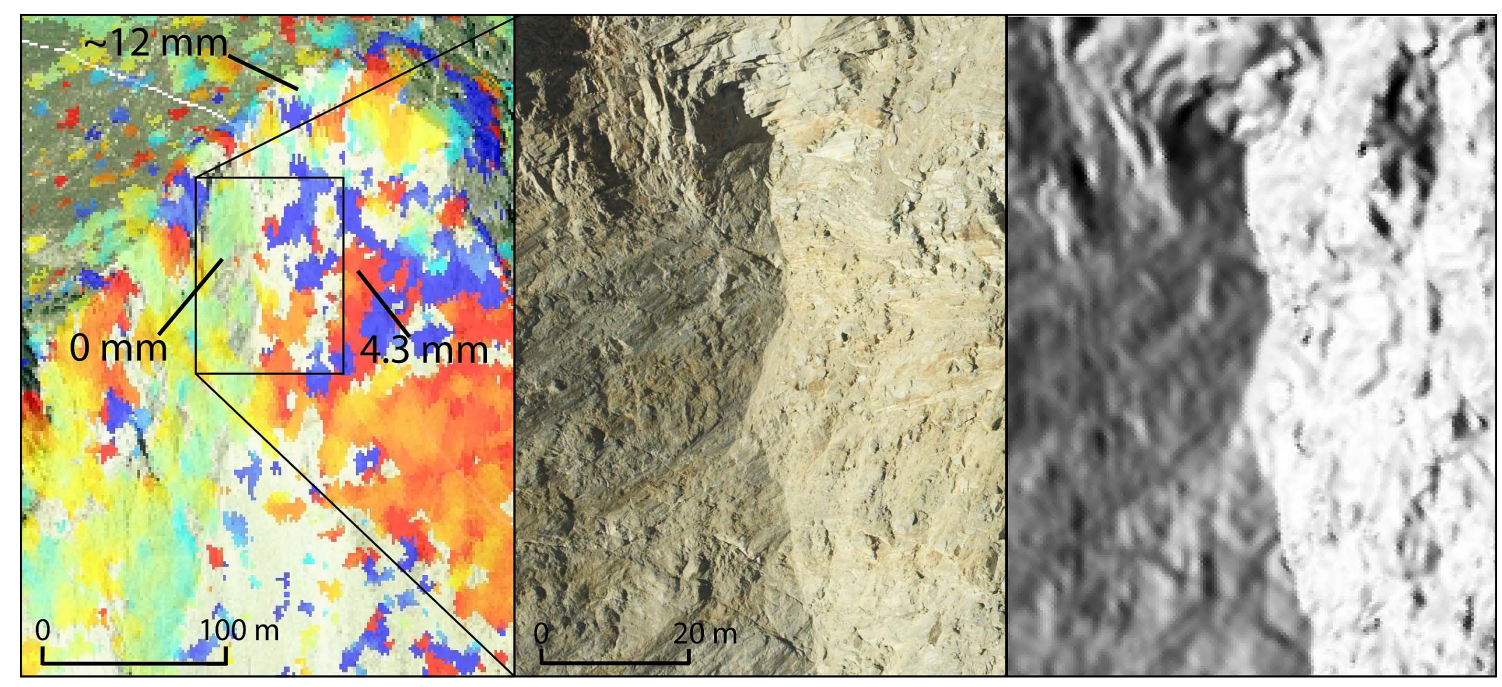

Fig. 5. GB-DInSAR displacement map (left), high resolution photo (middle), and shaded LiDAR DTM of the lateral release surface (oblique aerial view). This structure can be identified by the transition from $0 \mathrm{~mm}$ displacement on the left to more than $4 \mathrm{~mm}$ on the right. The unstable part on the right suffers from phase wrapping. On the photo and DTM, this transition can be seen as a wedge structure with a striated, smooth plane on the left and highly fractured rock on the right. The color scale for the GB-DInSAR image is the same as in Fig. 1. Location is shown in Fig. 2. 


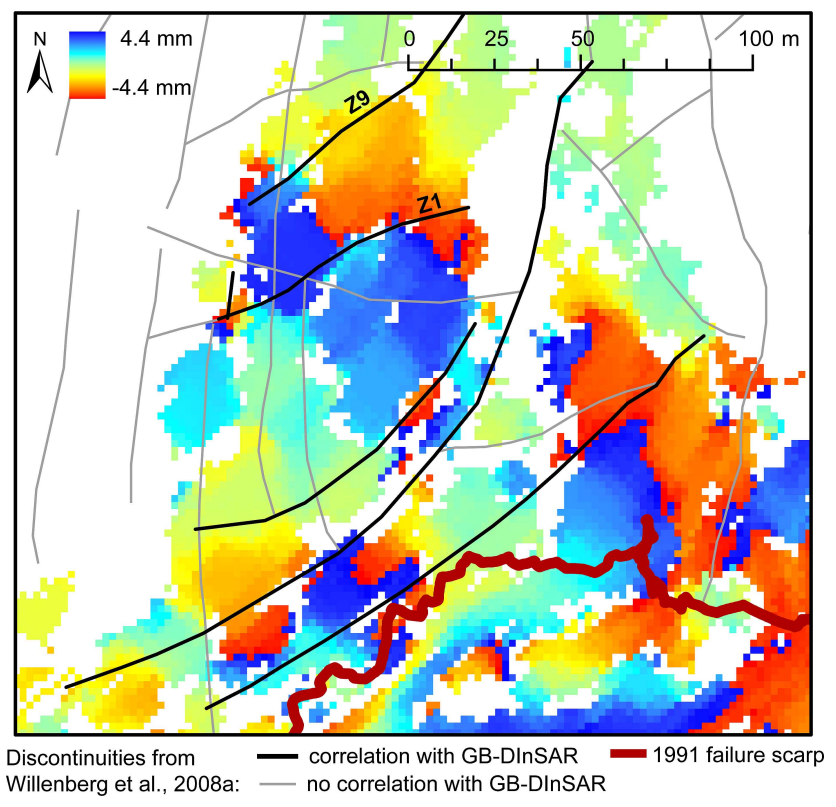

Fig. 6. Discontinuities mapped by Willenberg et al. (2008a) overlain on the GB-DInSAR displacement map representing 248 days of displacement. Although the displacement map is strongly affected by phase wrapping, some patterns clearly correlate with mapped discontinuities. For the discontinuities $\mathrm{Z} 1$ and $\mathrm{Z} 9$ opening rates were estimated using the GB-DInSAR displacements and compared to opening rates measured by hand. The GB-DInSAR derived opening rates gave similar but slightly lower values.

which are slightly lower than the opening rates from field measurements. Such discrepancies can be expected since the direction of relative displacement across these discontinuities deviates from the LOS of GB-DInSAR.

The GB-DInSAR displacement maps were also overlain with structures mapped on the $0.5 \mathrm{~m}$ LiDAR DTM from the inaccessible 1991 scarp surfaces in order to identify previously unmapped active discontinuities within the unstable rock mass. Figure 7 shows structures within the 1991 failure surface that correlate with GB-DInSAR displacement patterns in black, while structures not correlating with displacement patterns are shown in grey. Two local toppling instabilities superimposed on the large-scale unstable rock mass could be identified in photographs (inset in Fig. 7). In addition to the lateral release surface and the basal rupture plane, only a few structures within the unstable portion of the 1991 failure surface were found to correlate with GBDInSAR displacement patterns. Furthermore, the differential displacement along these structures is small with the exception of the local toppling instabilities. Thus, the large-scale movement of the rock mass is mainly controlled by the basal and the lateral release planes. Especially in the lower portion of the instability only little internal deformation/shearing occurs along large-scale discontinuities.

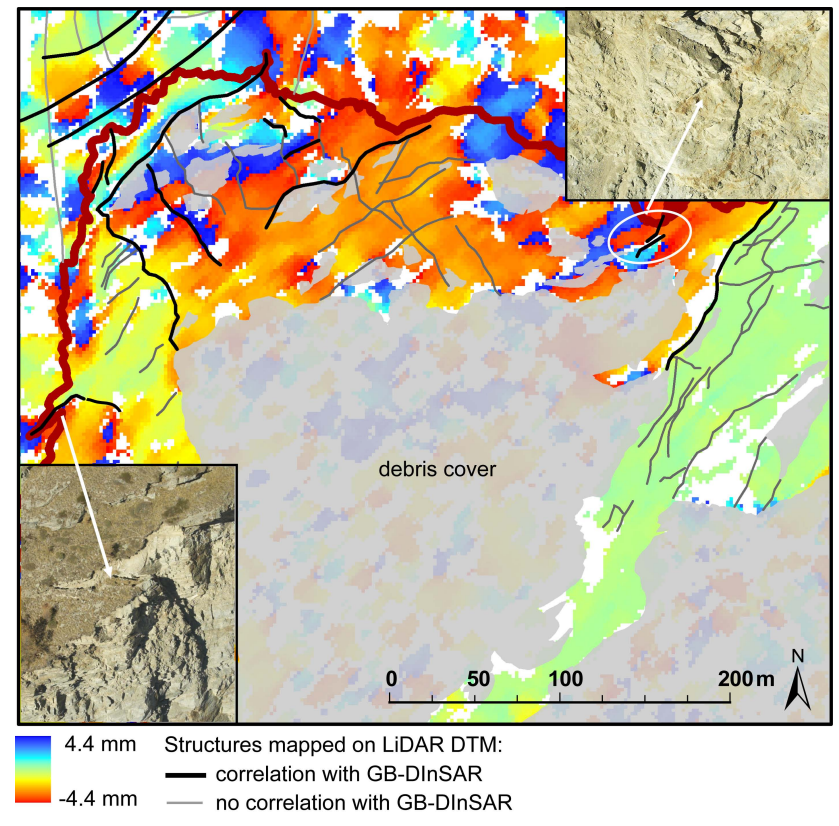

Fig. 7. The 248 days GB-DInSAR displacement map overlain by discontinuities mapped on the LiDAR DTM. Areas covered by debris are shaded in grey. Two local toppling instabilities could be identified and are highlighted in the inset photographs.

\subsection{Comparison of GB-DInSAR displacements with geodetic displacement measurements}

Since 1996, displacements of seven 3-D retro-reflectors were measured by surveying a geodetic network with a total station once or twice per year (Willenberg et al., 2008b; Jaboyedoff et al., 2004). The resultant vectors have an azimuth of $135-140^{\circ}$, which is sub-parallel to the LOS of the GB-DInSAR. Another 18 points were surveyed using a total station positioned on a monument close to the GBDInSAR base station resulting in a similar LOS. The total station surveys and the radar surveys were performed at times less than 50 days from each other. Displacement time series were derived from the five GB-DInSAR measurements at the locations of the geodetic reflectors by summing the displacements between each time interval. Figure 8 shows the time series for both GB-DInSAR and geodetic measurements, as well as the locations of these points on an unwrapped (i.e. phase wrapping removed) displacement map between the 2 nd and $3 r d$ repeat measurements (248 days interval). The error bars are $2.5 \mathrm{~mm}$ for the geodetic measurements and $1 \mathrm{~mm}$ for each GB-DInSAR repeat measurement. The latter error sums for each subsequent measurement. Comparison of the time series from both methods shows good agreement in most cases. However, in some instances GB-DInSAR shows lower velocities than the total station measurements (points 110, 130, and 151; Fig. 8), the average of these rate discrepancies is around $3 \mathrm{~mm} / \mathrm{yr}$. Time 


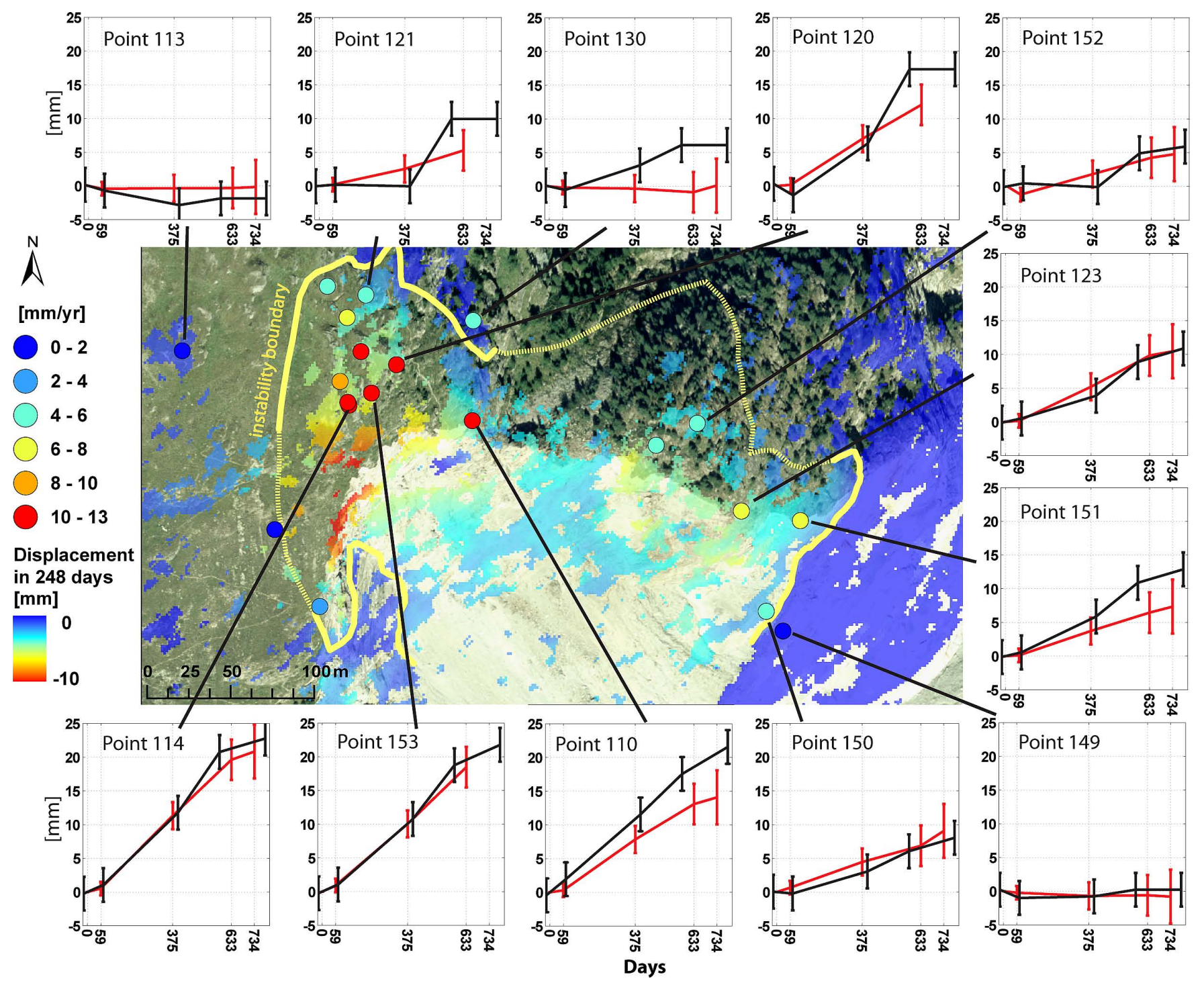

Fig. 8. Comparison of geodetic distance measurements with GB-DInSAR displacements. Time series derived from GB-DInSAR data at the locations of the reflectors are shown in red, geodetic time series are shown in black. Locations of the reflectors are displayed together with an unwrapped displacement map between the 3rd and the 2nd repeat survey ( 248 days). The yellow line shows the boundary of the instability derived from GB-DInSAR; dashed portions are uncertain.

series were also derived for points showing greater displacement than all geodetic points (red colors in the unwrapped Fig. 8). They show maximum velocities of about $20 \mathrm{~mm} / \mathrm{yr}$, which is greater than those measured with the 3-D geodetic network $(\sim 14 \mathrm{~mm} / \mathrm{yr})$.

\subsection{Comparison with micro-seismic activity}

Between 2002 and 2004, a seismic array consisting of 3 borehole geophones and 9 surface geophones recorded microseismic activity originating within the instability (Spillmann et al. 2007b). A probabilistic location algorithm taking into account a 3-D seismic velocity model was applied to the 223 events recorded by the array. The result of this proce- dure is a probabilistic density function (PDF) map, which shows areas of enhanced seismic activity (Fig. 9a). Willenberg et al. (2008b) pointed out that the seismic activity is generally distributed within the assumed instability boundaries, and that patches of high seismic activity tend to occur along mapped faults and fracture zones. The distribution of seismic activity partially correlates with the GB-DInSAR displacements map (Fig. 9b). The patches of high seismicity lie mostly within the mapped instability boundary as derived from GB-DInSAR. Furthermore, the areas with the highest displacement rates at the edge and top of the scarp correlate with an extended zone of enhanced seismic activity, which implies that the high displacements are accompanied 


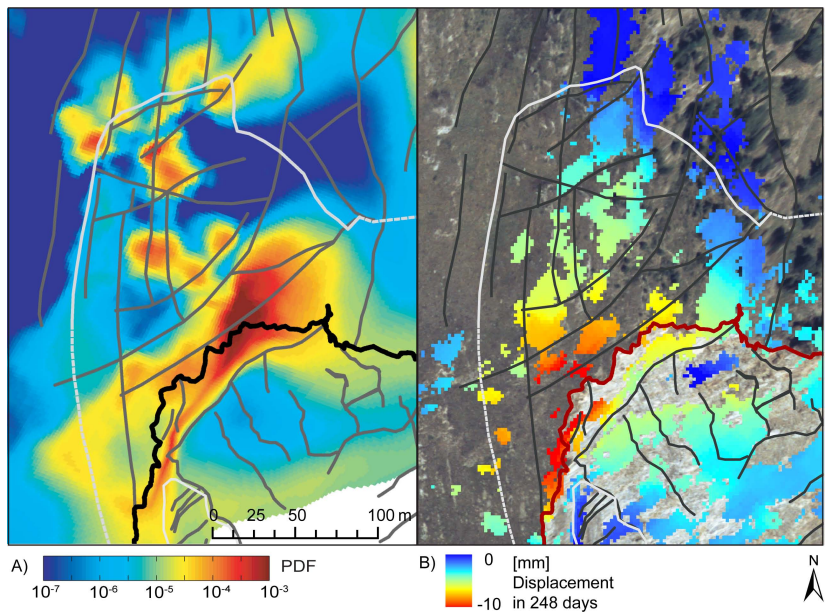

Fig. 9. (A) Micro-seismic activity map. The locations of 223 microseismic events recorded between 2002 and 2004 are represented as cumulative probabilistic density functions (PDF). High PDF values correspond to high micro-seismic activity. The values are representative for a surface at $15 \mathrm{~m}$ vertically below the topographic surface. (B) Unwrapped GB-DInSAR images as in Fig. 8. Both images include the boundary of the instability as derived from GB-DInSAR as well as the discontinuity map from Willenberg et al. (2008a).

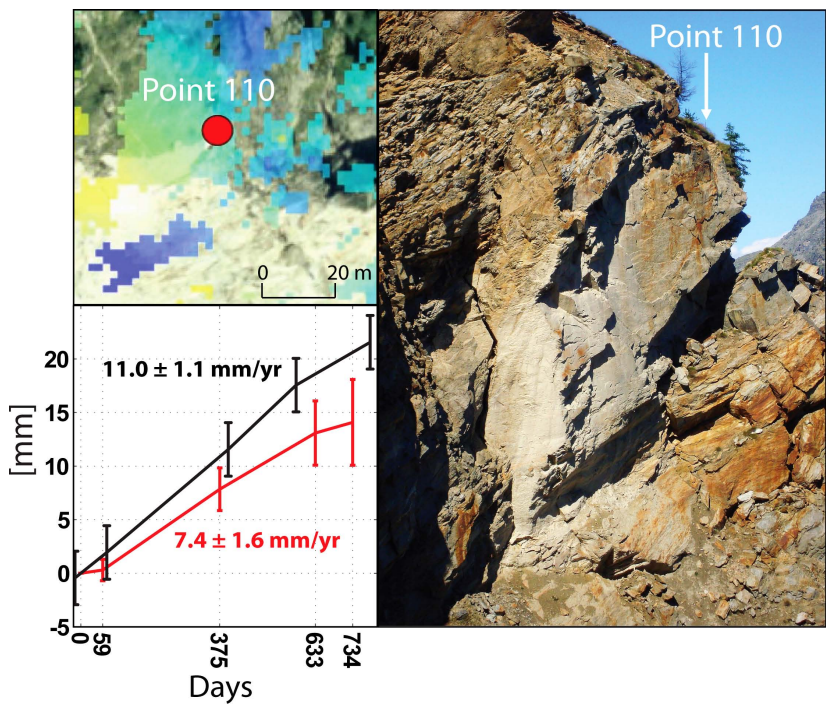

Fig. 10. Example of a geodetic point for which distance measurements (black line) yielded significantly higher velocities than those extracted from GB-DInSAR (red line). The reflector sits on a block bounded by large open cracks. Due to the size of the block its movements cannot be resolved with GB-DInSAR.

by strong internal deformation and shearing. The areas of low seismicity below correspond to the more uniformly moving areas on the GB-DInSAR maps (Fig. 1, region d). However, this region cannot be conclusively mapped as a low activity area, since the recorded seismicity may be adversely affected by high seismic attenuation within the strongly fractured rock mass.

\section{Discussion}

\subsection{Spatial resolution}

The spatial resolution of GB-DInSAR displacement maps is not only a function of the theoretical system resolution, but also influenced by post-processing filtering methods. Resolution is lowered by these filters as the signals are smeared over a few resolution cells (Tarchi et al., 2003a). Measured displacements are therefore representative of an extended area resulting in an effective resolution lower than the theoretical resolution given by the system properties (in our case $14 \times 14 \mathrm{~m}$ instead of $1.94-6.5 \mathrm{~m}$ ), and objects with high reflectivity contribute more to the measured values.

A comparison of mapped discontinuities and the GBDInSAR displacements (Fig. 6) shows that many discontinuities correlate with GB-DInSAR displacement patterns, although deviations of a few pixels occur. Opening rates across two discontinuities derived from GB-DInSAR are somewhat lower than field measurements. Such discrepancies can be caused by displacements oblique to the LOS and post-processing filters smearing displacements over a $14 \times 14 \mathrm{~m}$ area, although small errors in geo-referencing of the GB-DInSAR images can also introduce further discrepancies. Within the scarp created by the 1991 failures, only a few discontinuities were found to correlate with strong differential displacements on the GB-DInSAR maps. Limited resolution is a possible explanation for this observation, since sharp changes in the displacement pattern may be smoothed through filtering. However, it remains unknown whether such sharp differential displacement patterns are absent within the scarp due to filtering or because the real displacement field within the lower part of the current instability is continuous rather than localized along active structures. Continuous deformations could result from large-scale basal sliding, where large portions of the rock mass move at similar rates.

Resolution limitations can also explain discrepancies between displacement rates derived from GB-DInSAR and geodetic measurements. When a geodetic reflector moves at a different rate than the surrounding area because it is installed on a locally unstable block, the GB-DInSAR and geodetic measurements may not show the same displacements. Points 110, 130, and 151 (Fig. 8) show higher velocities for the total station than for the radar measurements, which likely results from blocks that move locally faster than the surrounding rock mass. An example is displayed in Fig. 10 for point 110. 


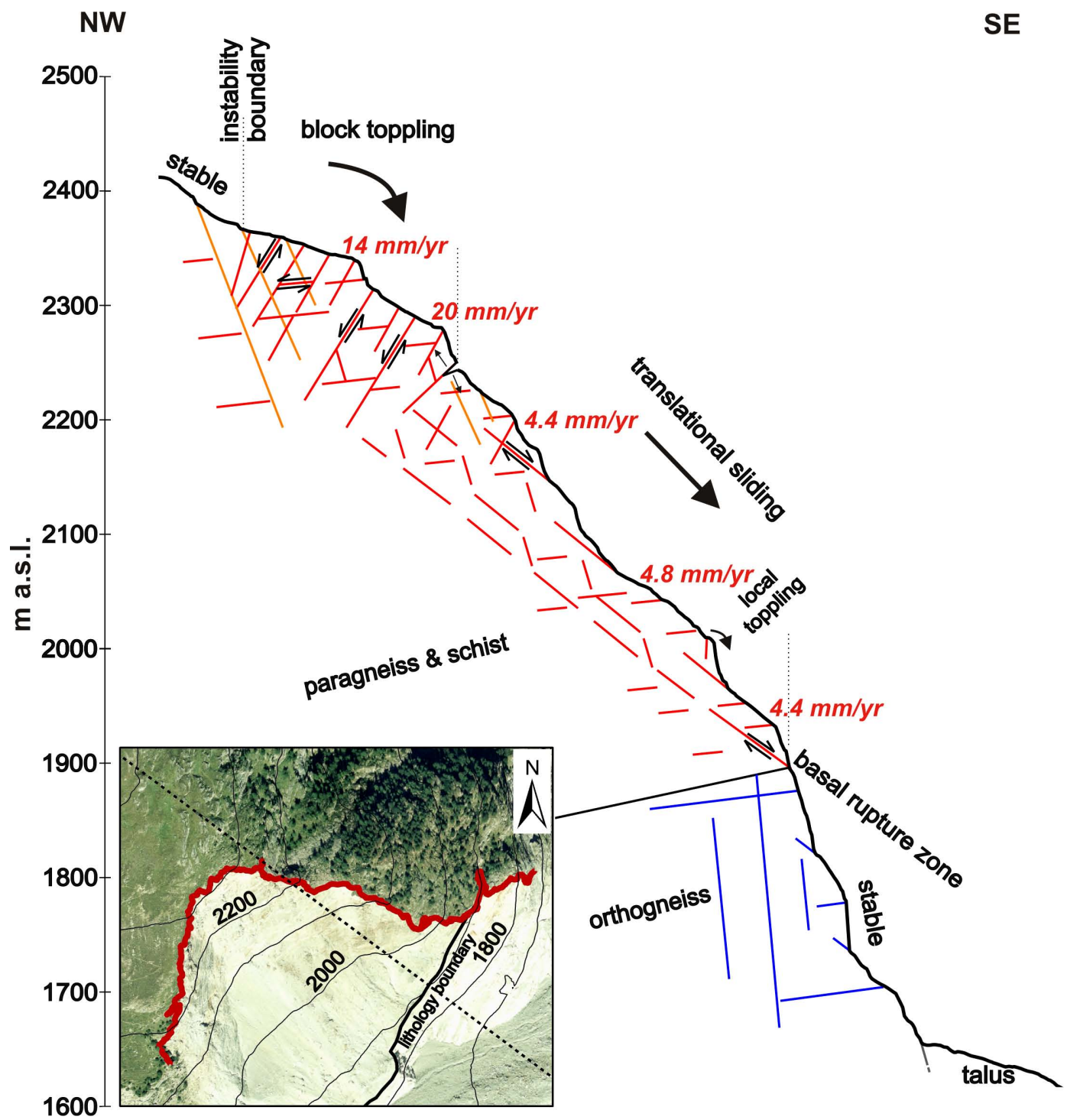

Fig. 11. Conceptual 2-D kinematic model of the instability. Both the results from Willenberg et al. (2008b) and from this study are included. The velocities indicated are the displacement rates derived from GB-DInSAR.

Such observations illustrate the spatial resolution limitations of GB-DInSAR, which are important to acknowledge in any attempt to interpret displacement patterns on a scale close to the effective resolution of the system. Differential displacements along active fractures can only be interpreted properly if their spatial extent and displacement difference is large enough to not be affected by resolution limits and filtering effects.

\subsection{Temporal resolution}

GB-DInSAR surveys at the slowly moving Randa rockslide showed that phase wrapping occurs when the time interval between repeat surveys was longer than about 100 days. This value may change with variable deformation rates ex- pected throughout the year. Phase unwrapping simplifies interpretation but introduces error and loss of data coverage (Fig. 8). For kinematic analysis it is essential to detect structural boundaries, such as a basal rupture zone, which requires a time interval sufficient to accumulate measurable deformation. Therefore, a trade-off exists between time intervals short enough to avoid phase wrapping and decorrelation, and long intervals that allow important structures to become distinguishable. Ideally, one would choose shorter time intervals and more repeat measurements. However, this is expensive in terms of both labor and cost. In our study, just four interferograms were available over a total period of 2 years. Nonetheless, individual images representing both short and long intervals provide complementary information. 


\subsection{Volume calculation}

Using the identified release planes, combined with mapped structures and the LiDAR DTM, we were able to define likely boundaries of the unstable rock mass at the Randa rockslide. We estimated the total volume to be $5.7 \pm 1.5$ million $\mathrm{m}^{3}$, which lies within the range given by Ischi et al. (1991). An orientation of 095/70 was used for the lateral release plane, while an orientation of $135 / 40$ for the basal shear zone was found to best match the trace observed with GB-DInSAR. This orientation also matches the orientation of the basal failure surface of the second 1991 event (Sartori et al., 2003) and the azimuth of geodetic displacement vectors. For the back boundary to the north-west a nearly vertical plane as given by Willenberg et al. (2008a) was assumed. The northern boundary of the instability remains uncertain since it lies in a partly shadowed and vegetated area with unreliable GB-DInSAR data. Two scenarios for the northern boundary were used to delineate the rock mass: 1 . the uninterrupted trace of the basal rupture zone daylighting to the north, or 2. a plane dipping steeply to the south and cutting the basal rupture surface at depth. The volume estimates for both scenarios differed by 0.6 million $\mathrm{m}^{3}$ for these two scenarios and were averaged for the final number.

\subsection{Implications for kinematics}

A preliminary analysis of the kinematics of the current instability can be deduced from displacement patterns. A 2-D conceptual model of the kinematics of the instability is displayed in Fig. 11, which combines results from both Willenberg et al. (2008b) and this study. The block toppling mechanism suggested for the top of the instability is confirmed by the GB-DInSAR images, which show a gradual increase of displacements from $4.4 \mathrm{~mm}$ to about $12 \mathrm{~mm}$ over a vertical distance of $\sim 150 \mathrm{~m}$ towards the top edge of the scarp (region (f) in Figs. 1 and 4). As shown on the micro-seismic activity maps by Spillmann et al. (2007b) in Fig. 9, internal deformation and shearing within this region are strongest, where the displacements rates are highest. Below the top of the scarp, a large area showing nearly uniform displacements (region (d) in Fig. 1c) suggests different kinematic behavior for the lower portion of the instability. This rather uniformly moving area has a displacement magnitude similar to that just above the basal rupture zone. Within this area only few sharp changes in the displacement field are found, as demonstrated in Fig. 7. Possibly only a few discontinuities are active within lower parts of the instability, whereas most of the deformation is localized along the basal rupture plane. We interpret this displacement pattern as translational movement on a planar or stepped shear surface, as hypothesized by Willenberg et al. (2008b), Jaboyedoff et al. (2004), and Sartori et al. (2003). The transition between toppling and translational failure occurs at a sharp change of the slope angle in the 1991 failure scarp from about $80^{\circ}$ at the top to about $60^{\circ}$ below. The displacement rate on the basal rupture surface is estimated to be $\sim 4.4 \mathrm{~mm} / \mathrm{yr}$. Some small-scale displacement patterns deviating from this uniform rate in this region are interpreted as superimposed secondary instabilities as shown in Fig. 7.

\section{Conclusions}

Displacements associated with a large-scale basal rupture zone and lateral release zone bounding the current instability at the Randa rockslide were detected using GBDInSAR. Structures associated with these displacements were confirmed with high resolution aerial photographs and a helicopter-based LiDAR DTM. The basal rupture zone is a highly persistent, narrow structure daylighting at the boundary between orthogneiss overlain by fractured paragneiss. Previous borehole surveys failed to intersect the basal rupture surface due to insufficient depth. The lateral release surface in the south was identified as a striated and steeplydipping fault, which can be regarded as the continuation of a lateral release surface from the 1991 rockslides. Both of these bounding structures outcrop on the inaccessible 1991 failure scarp and were not conclusively detected by previous investigations. The volume of the current instability was estimated to be $5.7 \pm 1.5$ million $\mathrm{m}^{3}$, and the area of the instability with maximum displacement rate (up to $\sim 20 \mathrm{~mm} / \mathrm{yr}$ ) was identified. Displacement patterns confirm block toppling in the upper part of the instability accompanied by microseismic activity suggesting some degree of internal deformation/shearing of the rock mass. The lower portion of the instability exhibits little differential displacement along discontinuities except for the basal rupture plane. Translational failure is thus suggested for lower regions of the instability as opposed to toppling at the top of the instability.

Many of the large discontinuities previously mapped within the accessible area at the top of the instability showed good correlation with displacement patterns on GB-DInSAR maps. In two cases, we could directly compare crack opening rates measured by hand and by GB-DInSAR, with good results. Comparison of GB-DInSAR displacements with total station measurements showed good agreement for most reflector points. However, three points showed higher velocities than measured with GB-DInSAR, which is likely due to local block movements. Such discrepancies also occur in the comparison of mapped structures with displacement patterns. They illustrate the influence of data filtering on the effective resolution of GB-DInSAR. For detailed interpretation of displacement patterns, such spatial resolution limitations of GB-DInSAR must be considered. 
Acknowledgements. GB-DInSAR surveys were performed by Ellegi Srl (Milano, Italy) using the GB-DInSAR LiSA system and LiSALab software; Carlo Rivolta provided technical support. GB-DInSAR measurements were financed by the Federal Office for the Environment, Switzerland. Geodetic measurements were conducted by Klaus Aufdenblatten (Zermatt, Switzerland) and directed by CREALP (Centre de Recherches sur 1'Environnement Alpin, Switzerland). Helimap Systems SA (Switzerland) performed the helicopter-based LiDAR survey. We thank Michel Jaboyedoff (University of Lausanne) and an anonymous reviewer for useful comments. This project was funded by the Swiss National Science Foundation (Project No. 2100-059238.99).

Edited by: A. Günther

Reviewed by: M. Jaboyedoff and another anonymous referee

\section{References}

Eberhardt, E., Stead, D., and Coggan, J. S.: Numerical analysis of initiation and progressive failure in natural rock slopes-the 1991 Randa rockslide, Int. J of Rock Mech. Min., 41(Eq. 1), 69-87, 2004.

Fortuny, J. and Sieber, A. J.: Fast Algorithm for a Near-Field Synthetic Aperture Radar Processor, IEEE T. Antenn. Propag., 42(10), 1458-1460, 1994.

Heincke, B., Green, A. G., van der Kruk, J., and Horstmeyer, H.: Acquisition and processing strategies for 3-D georadar surveying a region characterized by rugged topography, Geophysics, 70(6), K53-K61, 2005.

Heincke, B., Maurer, H., Green, A. G., Willenberg, H., Spillmann, T., and Burlini L.: Characterizing an unstable mountain slope using shallow 2-D and 3-D seismic tomography, Geophysics, 71(6), B241-B256, 2006.

Ischi, H., Keusen, H. R., and Scheller, E.: Randa Kt. Wallis. Bergsturz Grossufer vom April/Mai 1991, Zusammenfassender Bericht über die Aktivitäten der Geotest AG., unpublished report 91126 Geotest AG, Martigny, 1991.

Jaboyedoff, M., Ornstein, P., and Rouiller, J.-D.: Design of a geodetic database and associated tools for monitoring rock-slope movements: the example of the top of Randa rockfall scar, Nat. Hazards Earth Syst. Sci., 4, 187-196, 2004,

http://www.nat-hazards-earth-syst-sci.net/4/187/2004/.

Leva, D., Nico, G., Tarchi, D., Fortuny-Guasch, J., and Sieber, A. J.: Temporal Analysis of a Landslide by Means of a Ground-Based SAR Interferometer, IEEE T. Geosc. Remote, 41(4), 745-752, 2003.

Lingua, A., Piatti, D., and Rinaudo, F.: Remote monitoring of a landslide using an integration of GB-InSAR and LiDAR techniques, The International Archives of the Photogrammetry, Remote Sensing and Spatial Information, Sciences, XXXVII(B1), 361-366, 2008.
Lee, J.-S., Hoppel, K. W., Mango, S. A., and Miller, A. R.: Intensity and Phase Statistic of Multilook polarimetric and Interferomertric SAR Imagery, IEEE T., Geosc. Remote, 31(5), 1017-1028, 1994.

Loew, S., Willenberg, H., Spillmann, T., Heincke, B., Maurer, H. R., Eberhardt, E., and Evans, K.: Structure and kinematics of a large complex rockslide as determined from integrated geological and geophysical investigations (Randa, Switzerland), in: 1st North American Landslide Conference Association of Environmental and Engineering Geologists, edited by: Schaefer, V. R., Schuster, R. L., Turner, A. K., Vail, CO, 11 pp., 2007.

Sartori, M., Baillifard, F., Jaboyedoff, M., and Rouiller, J.-D.: Kinematics of the 1991 Randa rockslides (Valais, Switzerland), Nat. Hazards Earth Syst. Sci., 3, 423-433, 2003, http://www.nat-hazards-earth-syst-sci.net/3/423/2003/.

Schindler, C., Cuénod, Y., Eisenlohr, T., and Joris, C.-L.: Die Ereignisse vom 18. April und 9. Mai 1991 bei Randa (VS): ein atypischer Bergsturz in Raten, Eclogae Geol. Helv., 86(3), 643665, 1993.

Spillmann, T., Maurer, H., Willenberg, H., Evans, K. F., Heincke, B., and Green, A. G.: Characterization of an unstable rock mass based on borehole logs and diverse borehole radar data, J. Appl. Geophys., 61(Eq. 1), 16-38, 2007a.

Spillmann, T., Maurer, H., Green, A. G., Heincke, B., Willenberg, H., and Husen, S.: Microseismic investigation of an unstable mountain slope in the Swiss Alps, J. Geophys. Res., 112 (B07301), 2007b.

Tarchi, D., Casagli, N., Fanti, R., Leva, D., Luzi, G., Pasuto, A., Pieraccini, M., and Silvano, S.: Landslide monitoring by using ground-based SAR interferometry: an example of application to the Tessina landslide in Italy, Eng. Geol., 68, 15-30, 2003a.

Tarchi, D., Casagli, N., Moretti, S., Leva, D., and Sieber, A. J.: Monitoring landslide displacements by using ground-based synthetic aperture radar interferometry: Application to the Ruinon landslide in the Italian Alps, J. Geophys. Res., 108(B8), 2387, doi:10.1029/2002JB002204, 2003b.

Willenberg, H., Loew, S., Eberhardt, E., Evans, K. F., Spillmann, T., Heincke, B., Maurer, H., and Green, A. G.: Internal structure and deformation of an unstable crystalline rock mass above Randa (Switzerland): Part I - Internal structure from integrated geological and geophysical investigations, Eng. Geol., 101, 114, 2008a.

Willenberg, H., Evans, K. F., Eberhardt, E., Spillmann, T., and Loew, S.: Internal structure and deformation of an unstable crystalline rock mass above Randa (Switzerland): Part II Three-dimensional deformation patterns, Eng. Geol., 101, 1532, 2008 b. 